\title{
An analysis of tumor-related skin temperature differences in malignant soft-tissue tumors
}

\author{
Akiyoshi Shimatani ${ }^{1}{ }^{10} \cdot$ Manabu Hoshi $^{1} \cdot$ Naoto Oebisu $^{1} \cdot$ Naoki Takada $^{1} \cdot$ Yoshitaka Ban $^{1} \cdot$ Hiroaki Nakamura $^{1}$
}

Received: 2 August 2021 / Accepted: 28 September 2021 / Published online: 10 October 2021

(c) Japan Society of Clinical Oncology 2021, corrected publication 2021

\begin{abstract}
Background Soft tissue tumors are often accompanied with elevated skin temperature; however, studies concerning the relationship between soft-tissue tumors and skin temperature elevation are scarce. We aimed to evaluate the clinical significance of skin temperature elevation in soft-tissue tumors and identify factors related to skin temperature elevation.

Patients and methods This study comprised 118 patients at our hospital with soft-tissue tumors, excluding lipomatous tumors, whose pathological diagnosis was surgically confirmed between February 2017 and March 2021. Sixty-one and 57 patients had been diagnosed with benign lesions and malignant tumors, respectively (men, 64; women, 54; median age, 61 [range, 20-92] years). The relationship between skin temperature, monitored using a thermography camera, and the presence of soft-tissue malignancy was investigated. We reviewed clinical data to identify factors related to elevated skin temperature. Results Temperature differences $\geq 0.2{ }^{\circ} \mathrm{C}$ compared to the unaffected side were significantly associated with the presence of malignant tumors $(p<0.001)$. Logistic regression analysis indicated that intertumoral blood supply was associated with elevated skin temperature (OR 3.22, 95\% CI 2.03-5.13; $p<0.001$ ).

Conclusions Elevated skin temperature, influenced by intertumoral blood supply, may be an important adjunct to physical findings when diagnosing malignant soft-tissue tumors.

Clinical relevance Intertumoral blood supply influenced elevated skin temperature in malignant soft-tissue tumors. A skin temperature difference $\geq 0.2^{\circ} \mathrm{C}$ compared to the unaffected side can help differentiate between benign and malignant tumors. Skin temperature differences may help in diagnosing malignant soft-tissue tumors.
\end{abstract}

Keywords Soft tissue tumor $\cdot$ Skin temperature $\cdot$ Thermography $\cdot$ Cancer screening $\cdot$ Blood supply

\section{Introduction}

On palpation, skin temperature is an important clinical indicator when physically examining soft-tissue tumors, often accompanied by elevated skin temperature. Previous studies have reported that malignant tumors, such as melanoma and breast cancer tumors, present with skin temperature higher than surrounding healthy tissue [1-3]. However, benign skin lesions, such as cysts, dermatofibromas, granulomas, seborrheic keratoses, and epidermal inclusion cysts, are iso- or hypothermic compared with the surrounding skin [2, 3]. Differences in heat generation between malignant and healthy

Akiyoshi Shimatani

bpcip920@tcct.zaq.ne.jp

1 Department of Orthopedic Surgery, Osaka City University Graduate School of Medicine, 1-4-3 Asahi-Machi, Abeno-ku, Osaka 545-8585, Japan tissues have been attributed to differences in blood supply, metabolic rate, and the extent of angiogenesis [4-7]. However, studies on the relationship between soft-tissue tumors and elevated skin temperature are scarce [8].

A significant association between elevated skin temperature and the presence of malignancy has previously been reported in a study of 100 patients treated for soft-tissue tumors [8]. However, of these, 42 patients with lipoma and well-differentiated liposarcoma had low skin temperatures. Thus, the presence of malignant tumors, excluding adipose tumors, may have contributed to increased skin temperature. However, identifying clinical factors concerning tumors that elevate the skin temperature, which might help the histological diagnosis, has not previously been investigated [8]. Therefore, studies with large sample size, excluding adipose tumors, are necessary to identify factors that affect skin temperature. 
Thermography, also known as digital infrared thermal imaging, is one of the most prevalent diagnostic methods developed to investigate disorders related to skin temperature. This non-invasive, safe, and low-cost technique measures the temperature of infrared rays emitted from the body $[9,10]$, and can be used to diagnose several cancers through detecting tumor-related skin temperature increases $[11,12]$. Other applications of this technique include diabetes treatment monitoring [13] and detecting COVID-19 [14].

This study aimed to determine the clinical significance of temperature elevation in soft-tissue tumors by assessing the difference in skin temperature elevation between benign lesions and malignant tumors using thermography and identifying the relationship between skin temperature and the presence of malignancy. Furthermore, we aimed to identify factors related to increased skin temperature using clinical data.

\section{Patients and methods}

\section{Patients}

This retrospective study comprised 204 patients with softtissue tumors, with a histological diagnosis confirmed by surgery, at our institution between February 2017 and March 2021. In this study, we included all patients with relevant measured and recorded clinical data. Exclusion criteria comprised patients aged $<20$ years, those diagnosed with lipoma and well-differentiated liposarcoma that show a low skin temperature based on a previous study [8], and patients with incomplete clinical data. In total, 118 patients (men, 64; women, 54) were selected (Fig. 1) for inclusion in our study [median age at diagnosis, 61 (range 20-92) years]. Of these, 61 patients had benign lesions, and 57 patients had malignant tumors (Table 1). General characteristics, including tumor depth (superficial or deep), location, and histological grade were recorded for each patient (Table1). Whether the tumor was superficial or deep was judged using the fascia as the boundary. After surgical removal or biopsy, all soft-tissue tumors were diagnosed according to the World Health Organization (WHO) histological classification by experienced pathologists specialized in soft-tissue cancer [15]. The histological tumor grade, which was determined using the French Federation of Cancer Centers Sarcoma Group system for 51 malignant soft-tissue tumors other than the 6 non-sarcomas, was reviewed and confirmed by independent pathologists. This study included a considerable number of cases of non-neoplastic benign soft-tissue tumorlike and malignant non-sarcoma lesions, such as epidermal cyst ( 5 cases), crystal deposition ( 2 cases), bursitis (1 case), endometriosis ( 1 case), fat necrosis ( 1 case), adenocarcinoma ( 2 cases), endometrioid carcinoma ( 1 case), malignant lymphoma ( 1 case), Merkel cell carcinoma (1 case), and thyroid cancer (1 case) (Table 1). This is because the lesions had a histologically definitive diagnosis after surgery and were treated as soft-tissue tumors on preoperative imaging. At the initial visit, it was not easy to distinguish malignant and soft-tissue tumors, including non-soft-tissue tumors and inflammatory masses, even with physical examination. Since this study is related to the preoperative diagnosis of soft-tissue tumors, these cases were included. This study was approved by our Institutional Review Board. Informed consent was obtained from all patients after a full surgical explanation had been provided.

\section{Tumor-related skin temperature and pathological diagnosis}

Actual skin temperatures concerning affected and unaffected regions were measured and visualized using a thermography camera (C3 thermal camera [FLIR ${ }^{\circledR}$, USA], Fig. 2a, b). The recorded thermograms were analyzed using the cameras' corresponding software. The exposed region of interest (ROI) was defined as the area encompassing the entire tumor on the affected side and the area symmetrical to the affected side on the unaffected side. As every participant had a different tumor location, the area of the selected ROIs could not be equal. However, the ROI on the affected and unaffected sides of an individual could be the same. On the affected side, the skin temperature just above the center of the tumor was measured at three points as much as possible in the recorded thermogram, and the average of these measurements was calculated. The same measurements were performed at the three corresponding points on the unaffected side. To ensure data reliability, using the same 20 affected thermograms, the skin temperature at the center of the tumor was measured and recorded by three examiners at different times. The inter-observer reliability was verified using these data. Skin temperature difference was defined as the difference between the average skin temperature at the affected and unaffected regions. For all 118 patients, skin temperature differences were recorded (Table 1). We compared differences in skin temperature values between benign lesions and malignant tumors. Receiver-operating characteristic (ROC) curve analyses were conducted to determine the skin temperature difference thresholds between affected and unaffected tissues to differentiate between benign lesions and malignant tumors.

\section{The relationship between tumor-related skin temperature and clinical data}

We investigated the relationship between tumor-related skin temperature monitored using a thermography camera and patients' clinical data regarding benign or malignant 
Fig. 1 A flow diagram of patient inclusion in the study. Of 204 patients, 86 were excluded. Of these, 6 were aged $<20$ years, 52 had a histological diagnosis of lipoma or well-differentiated liposarcoma, and 28 had incomplete clinical data. In total, 118 patients were included in this study. Of these, 26 patients had undergone PET-CT imaging scans prior to surgery and SUVmax values had been recorded

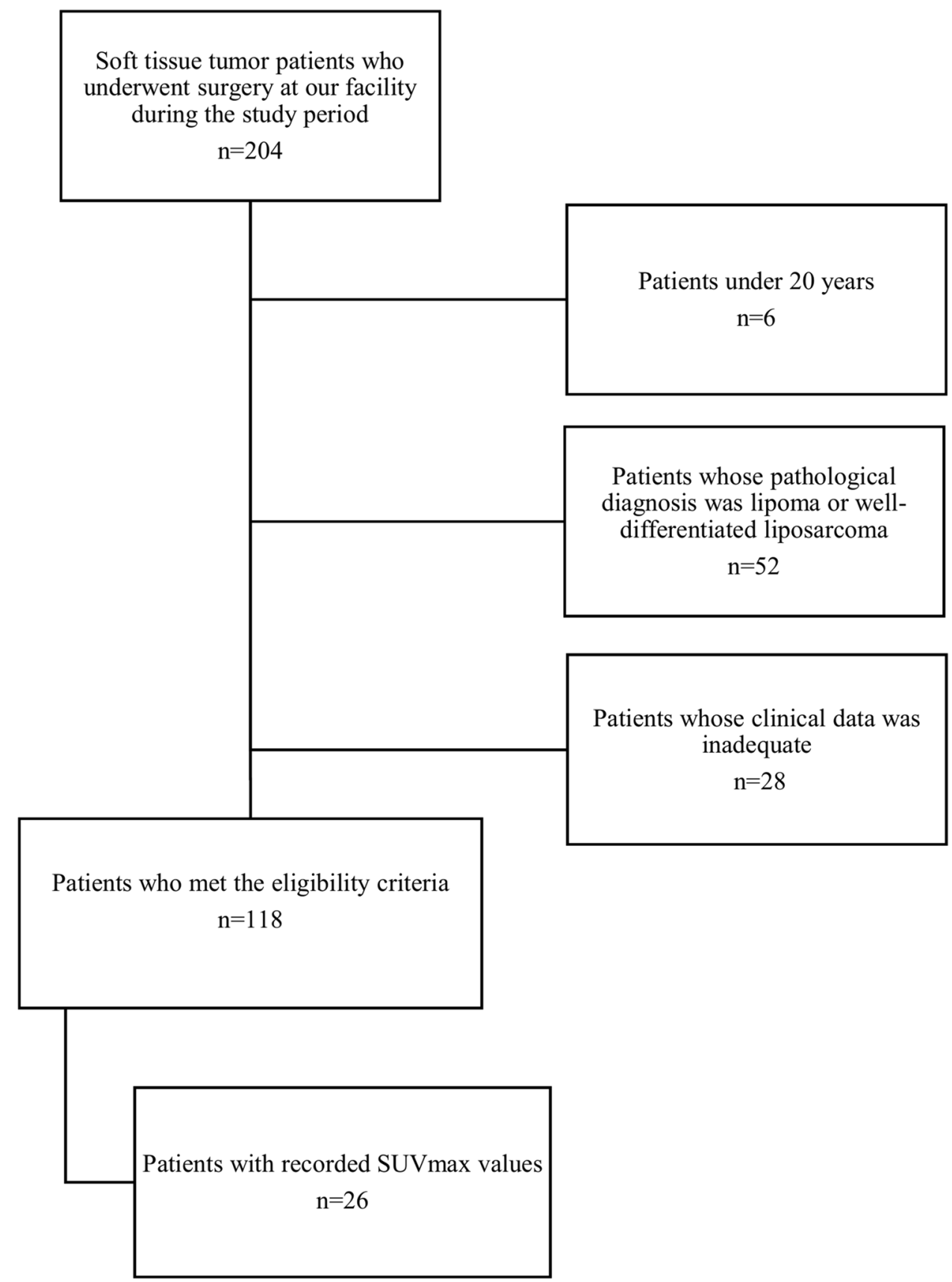

soft-tissue tumors. The clinical data assessed were sex, age, tumor diameter, tumor distance, C-reactive protein (CRP) levels, the amount of intertumoral blood supply, and histological grade. The clinical data other than histological grade were verified for all 118 patients. Tumor diameter and distance were measured using preoperative magnetic resonance imaging (MRI). Tumor diameter was recorded along the major axis, and tumor distance was recorded as the shortest distance from the skin to the edge of the tumor. Patients' preoperative CRP levels had been obtained prior to treatment, and, in this study, we used the last obtained CRP levels in our analysis. The amount of intertumoral blood supply was evaluated preoperatively in the operating room using color Doppler ultrasonography (Hitachi-Aloka Medical, Tokyo, Japan). According to the inflow of blood vessels, tumors were classified into four grades using the modified Giovagnorio classification, hereafter referred to as $\mathrm{G}$ grade; grade 1, no blood supply in the tumor; grade 2, 1-2 blood vessels providing inflow to the tumor; grade 3, a large number of vascular inflows into the tumor; and grade 4, an abundant blood supply inside the tumor (Fig. 2c) [16]. 
Table 1 Patient characteristics and skin temperature differences

\begin{tabular}{|c|c|c|c|}
\hline Characteristics & Number & $\begin{array}{l}\text { Mean of temperature dif- } \\
\text { ferences }\left({ }^{\circ} \mathrm{C}\right)\end{array}$ & SD \\
\hline Total patients & 118 & & \\
\hline Age (years) & $61(20-92)$ & & \\
\hline \multicolumn{4}{|l|}{ Sex } \\
\hline Male & 64 & & \\
\hline Female & 54 & & \\
\hline \multicolumn{4}{|l|}{ Histology } \\
\hline Benign & 61 & 0.03 & 0.26 \\
\hline Schwannoma & 26 & 0.08 & 0.24 \\
\hline GCTTS & 6 & 0.07 & 0.22 \\
\hline Hemangioma & 6 & 0.07 & 0.15 \\
\hline Epiermal cyst & 5 & -0.24 & 0.19 \\
\hline Desmoid & 3 & -0.03 & 0.06 \\
\hline Myxoma & 3 & -0.03 & 0.06 \\
\hline Synovial osteochondromatosis & 3 & -0.07 & 0.12 \\
\hline Crystal deposition & 2 & 0.4 & 0.4 \\
\hline Angioleioma & 1 & -0.4 & \\
\hline Bursitis & 1 & 0 & \\
\hline Cellular angiofibroma & 1 & 0.7 & \\
\hline Endometriosis & 1 & 0.1 & \\
\hline Fat necrosis & 1 & 0.1 & \\
\hline Fibroma & 1 & 0 & \\
\hline Mixed tumor & 1 & -0.1 & \\
\hline Malignant & 57 & 0.35 & 0.42 \\
\hline Undifferentiated pleomorphic sarcoma & 8 & 0.36 & 0.37 \\
\hline Dedifferentiated liposarcoma & 6 & 0.18 & 0.28 \\
\hline Myxofibrosarcoma & 6 & 0.27 & 0.36 \\
\hline MPNST & 5 & 0.32 & 0.26 \\
\hline Myxoid liposarcoma & 4 & 0.28 & 0.38 \\
\hline Low grade fibromyxoid sarcoma & 3 & 0.13 & 0.35 \\
\hline Adenocarcinoma & 2 & 0.1 & 0.14 \\
\hline Angiosarcoma & 2 & 1.2 & 0.42 \\
\hline Fibrosarcoma & 2 & 0.6 & 0.85 \\
\hline Leiomyosarcoma & 2 & 0.2 & 0.42 \\
\hline Myxoid fibrosarcoma & 2 & 0.15 & 0.21 \\
\hline Plemorphic liposarcoma & 2 & 0.55 & 0.07 \\
\hline Solitary fibrous tumor & 2 & 0.55 & 1.2 \\
\hline Endometrioid carcinoma & 1 & 0.3 & \\
\hline Ewing sarcoma & 1 & 0.3 & \\
\hline Gramulocytric sarcoma & 1 & 0.3 & \\
\hline Malignant Lymphoma & 1 & 0.2 & \\
\hline Malignant GCT & 1 & 0 & \\
\hline Merkel cell carcinoma & 1 & 1.7 & \\
\hline Osteosarcoma & 1 & 0.4 & \\
\hline Perivascular epithelioid cell tumor & 1 & 0.3 & \\
\hline Plemorphic rhabdomyosarcoma & 1 & 0.2 & \\
\hline Synovial sarcoma & 1 & 0.3 & \\
\hline Thyroid cancer & 1 & 0.4 & \\
\hline \multicolumn{4}{|l|}{ Depth } \\
\hline Superficial & 61 & 0.26 & 0.46 \\
\hline Deep & 57 & 0.11 & 0.25 \\
\hline
\end{tabular}


Table 1 (continued)

\begin{tabular}{llll}
\hline Characteristics & Number & $\begin{array}{l}\text { Mean of temperature dif- } \\
\text { ferences }\left({ }^{\circ} \mathrm{C}\right)\end{array}$ \\
\hline Tumor location & 22 & \\
$\quad$ Trunk & 13 & \\
$\quad$ Upper arm & 17 & & \\
Forearm & 34 & & \\
Thigh & 32 & 0.26 & 0.54 \\
Leg & & 0.35 & 0.39 \\
Histological grade & 12 & 0.36 & 0.07 \\
1 & 22 & 0.47 & 0.25 \\
2 & 17 & 6 & \\
3 & 6 & & \\
Non sarcoma & & & \\
\hline
\end{tabular}

GCT giant cell tumor; GCTTS giant cell tumor of the tendon sheath; MPNST malignant peripheral nerve sheath tumor; $S D$ standard deviation

Fig. 2 Images of a representative case. a A malignant soft-tissue tumor in the right thigh was diagnosed as myxofibrosarcoma. The white and red shadow indicates a higher temperature relative to the surrounding tissues. b Sections of the myxofibrosarcoma stained with haematoxylin and eosin (magnification: $\times 200$ ). Polynuclear large cells or polymorphic cells proliferating irregularly against a background of mucous substrate is observed. c An echo-color Doppler image. The tumor blood supply was classified as grade 4, based on the abundant blood supply observed within the tumor

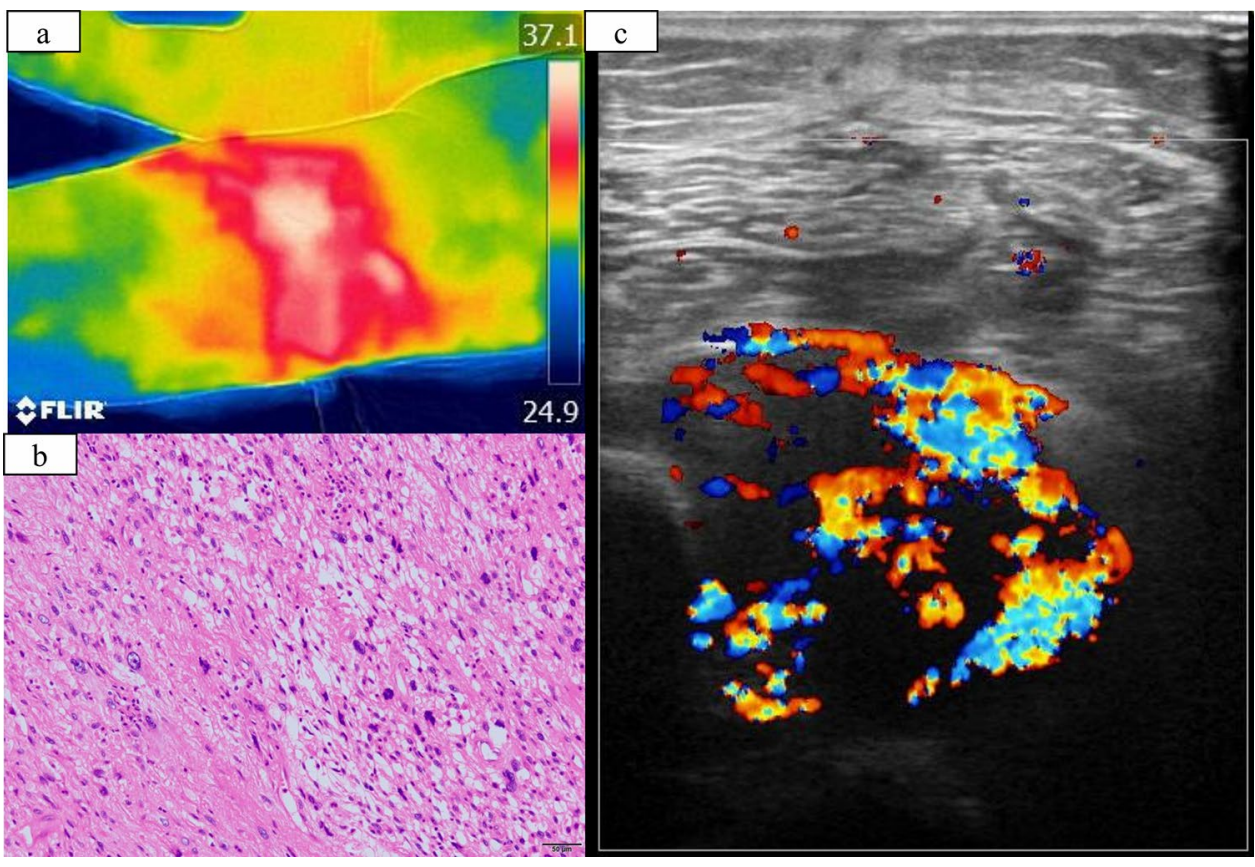

\section{The relationship between tumor-related skin temperature elevation and glucose metabolism}

We investigated the relationship between the maximum standardized uptake value (SUVmax) of the tumor and the difference in skin temperature. Of 118 patients, 26 patients had undergone integrated 2-deoxy-2- $\mathrm{F}^{18}$-fluoro-D-glucose positron emission tomography combined with computed tomography (FDG-PET-CT) scans, which had been taken prior to surgery, and SUVmax values had been recorded (Fig. 1). CT and PET images were routinely acquired from the orbit to the proximal thigh $60 \mathrm{~min}$ after intravenous injection of $2.7 \mathrm{MBq} / \mathrm{kg}$ of FDG. If necessary, additional images of the toes were captured. PET scans were performed using a whole-body PET/CT scanner (Discovery ST; GE Healthcare, Tokyo, Japan).

\section{Statistical analysis}

Inter-observer reliability was determined by calculation of the Intraclass Correlation Coefficient (ICC) with 95\% confidence intervals (CI) [17]. Student's t- and Fisher's exact tests were used to compare the two groups. ROC curve analysis was used to determine the cut-off value of the skin temperature difference between affected and unaffected tissues to distinguish benign from malignant tumors. A logistic 
regression model was used to calculate the adjusted odds ratio (OR) with a 95\% confidence interval (CI) to elucidate the factors associated with elevated skin temperature. The study's objective variable was a cut-off value for the difference in skin temperature between benign and malignant tumors. Factors included in the multivariate model were sex (male, female), age (1-year increments), tumor size $(0.1-\mathrm{cm}$ increments), tumor distance (in 0.01-mm increments), CRP level $(0.01-\mathrm{mg} / \mathrm{dL}$ increments), and $\mathrm{G}$ grade (1-grade increments). The level of significance was set at $p=0.05$. Statistical analysis was performed using Excel Statistical Software for Windows (version 2020; SSRI Co. Ltd., Tokyo, Japan).

\section{Results}

\section{Tumor-related skin temperature and pathological diagnosis}

The value of ICC was 0.9982 (95\% CI 0.9962-0.9992) $(p<0.001)$. Table 2 shows the clinical parameters of benign lesions and malignant tumors. Benign lesions were diagnosed in 30 men and 31 women, and malignant tumors were diagnosed in 34 men and 23 women. The average values \pm standard deviation (SD) for age, tumor size, tumor distance, and CRP levels in benign lesions were $56.9 \pm 17.4$ years, $4.3 \pm 2.9 \mathrm{~cm}, 9.3 \pm 10.0 \mathrm{~mm}$, $0.1 \mathrm{mg} / \mathrm{dL}( \pm 0.1 \mathrm{mg} / \mathrm{dL})$, respectively. Concerning malignant tumors, these values were $66.0 \pm 14.1$ years, $7.7 \pm 5.7 \mathrm{~cm}, 6.2 \pm 6.9 \mathrm{~mm}, 1.0 \pm 2.6 \mathrm{mg} / \mathrm{dL}$, respectively. The median (inter-quartile range [IQR]) $\mathrm{G}$ grade value in benign lesions was 2 (range 1-3), and in malignant tumors, the median $\mathrm{G}$ grade value was 3 (range 2-4). Malignant tumors were statistically significantly larger in size $(p<0.001)$, had higher CRP levels $(p=0.02)$, and had a higher intertumoral blood supply than benign lesions

Table 2 Relationship between clinical data and pathological diagnosis

\begin{tabular}{lllc}
\hline & Benign & Malignant & $p$ value \\
\hline Sex $(n)$ & & & 0.27 \\
$\quad$ Men & 30 & 34 & \\
Women & 31 & 23 & \\
Age (years old) & $56.9 \pm 17.4$ & $66.0 \pm 14.1$ & $0.0024^{*}$ \\
Size (cm) & $4.3 \pm 2.9$ & $7.7 \pm 5.7$ & $<0.001^{*}$ \\
Distance (mm) & $9.3 \pm 10.1$ & $6.2 \pm 6.9$ & 0.06 \\
CRP (mg/dL) & $0.1 \pm 0.1$ & $1.0 \pm 2.6$ & $0.02^{*}$ \\
G grade (median [IQR]) & $2[1-3]$ & $3[2-4]$ & $<0.001^{*}$ \\
\hline
\end{tabular}

CRP C-reactive protein, $G$ grade grades (1-4) classified according to the modified Giovagnorio classification based on the amount of blood supply in the tumor, IQR inter-quartile range

$* p<0.05$ $(p<0.001)$. In contrast, tumor distance was not found to be statistically significantly relevant $(p=0.06)$. Skin temperature differences for each patient are shown in Table 1. The average \pm SD skin temperature differences in patients with benign lesions was $0.03 \pm 0.26{ }^{\circ} \mathrm{C}$, whereas, in patients with malignant tumors, the average \pm SD skin temperature was $0.35 \pm 0.42{ }^{\circ} \mathrm{C}$ (Fig. 3).

According to ROC curve analysis, the cut-off value of skin temperature differences in benign lesions and malignant tumors was $0.2{ }^{\circ} \mathrm{C}$, the area under the curve (AUC) was 0.75 , and the OR was 6.71 (sensitivity $66.7 \%$; specificity $77.0 \%$; Fig. 4). Fourteen of 61 patients with benign lesions and 38 of 57 patients with malignant tumors had higher lesion skin temperature values of at least $0.2{ }^{\circ} \mathrm{C}$ compared with unaffected tissues (Table 3 ). Skin temperature differences $\geq 0.2{ }^{\circ} \mathrm{C}$ were significantly associated with the presence of malignant tumors $(p<0.001)$.

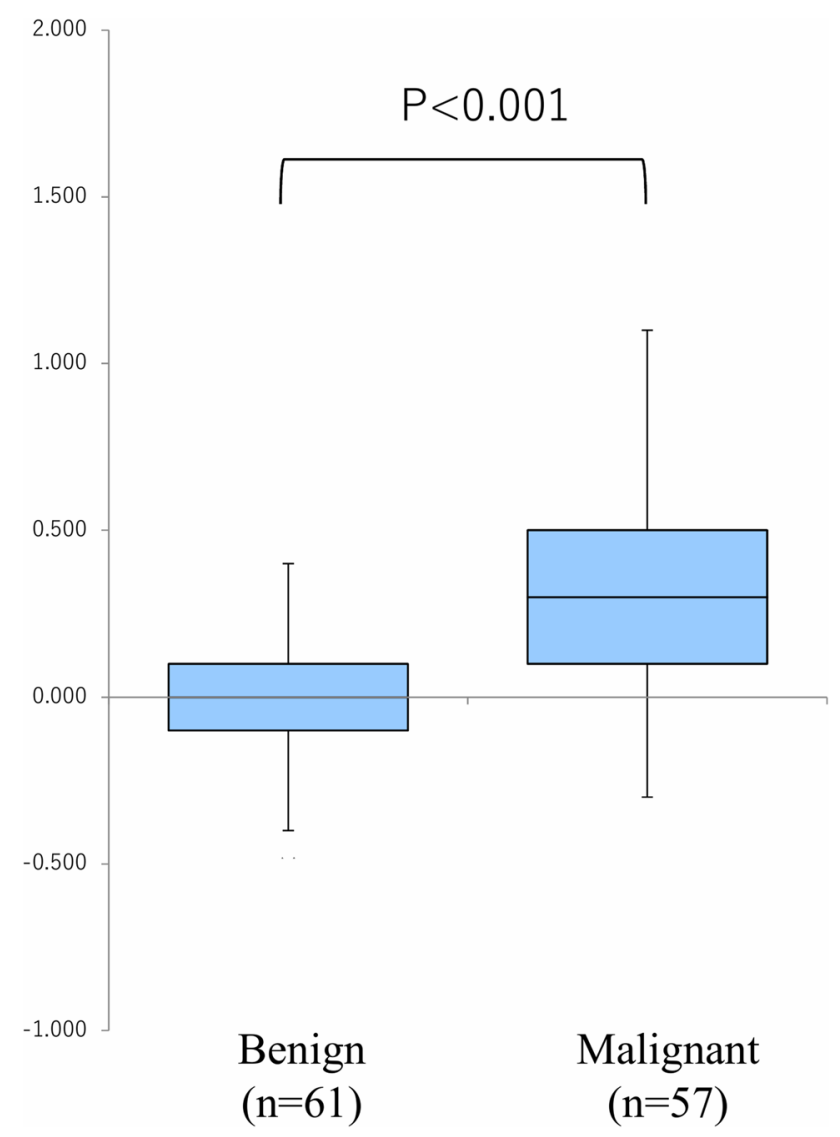

Fig. 3 A comparison of skin temperature differences between benign lesions and malignant tumors. The average \pm standard deviation skin temperature difference in benign lesions $(n=61)$ was $0.03 \pm 0.26^{\circ} \mathrm{C}$. In malignant tumors $(n=57)$, the average \pm standard deviation skin temperature difference was $0.35 \pm 0.42{ }^{\circ} \mathrm{C}$. The skin temperature difference in malignant tumors was found to be statistically significantly higher than that of benign lesions $(p<0.001)$. The vertical axis shows the value of the difference in skin temperature 


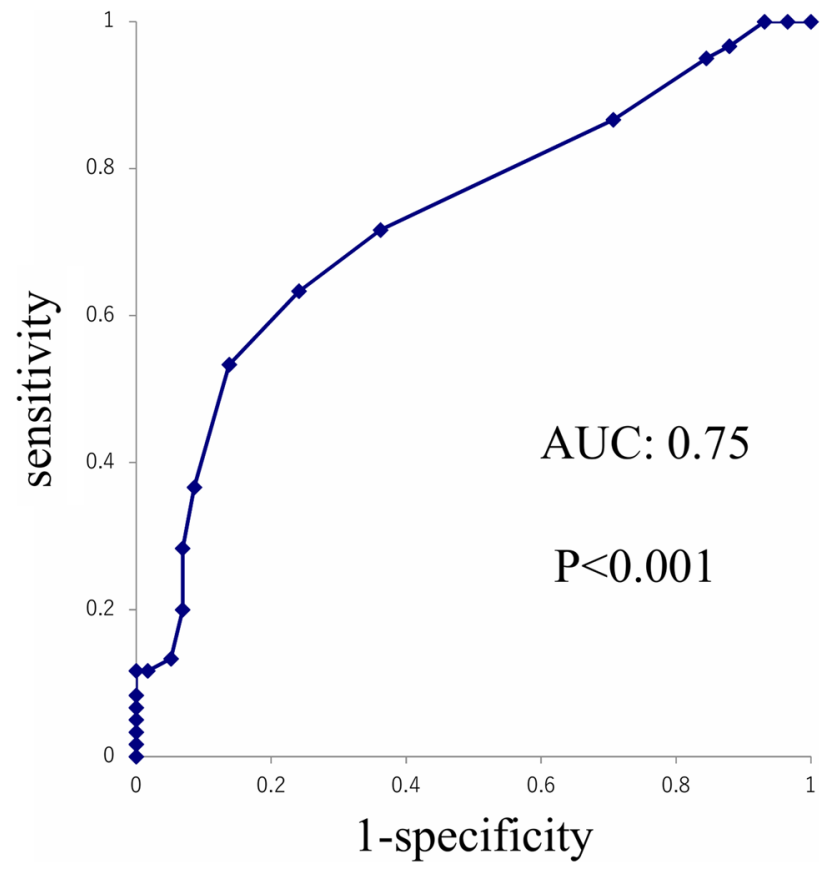

Fig. 4 Receiver-operating characteristic curve analysis of skin temperature differences between benign and malignant tumors. The cutoff value for the difference in skin temperature was $0.2{ }^{\circ} \mathrm{C}$. The area under the curve was 0.75 , and the odds ratio, sensitivity, and specificity were $6.71,0.67$, and 0.77 , respectively

Table 3 Pathological diagnosis and actual skin temperature differences

\begin{tabular}{llll}
\hline & \multicolumn{2}{l}{ Skin temperature differences $\left({ }^{\circ} \mathrm{C}\right)$} & Total \\
\cline { 2 - 3 } & $0.2 \leq$ & $<0.2$ & \\
\hline Malignant $(n)$ & 38 & 19 & 57 \\
Benign $(n)$ & 14 & 47 & 61 \\
Total $(n)$ & 52 & 66 & 118 \\
\hline
\end{tabular}

\section{The relationship between tumor-related skin temperature and the clinical parameters}

Table 4 shows the relationship between tumor-related skin temperature and the clinical parameters. There were 52 softtissue tumors (men, 28; women, 24) with a skin temperature difference $>0.2^{\circ} \mathrm{C}$ and 66 (men, 37; women, 29) with a skin temperature difference $<0.2{ }^{\circ} \mathrm{C}$. The average age $\pm \mathrm{SD}$, tumor size, tumor distance, CRP levels, and median values (IQR) for $\mathrm{G}$ grade tumors and histological grade with a skin temperature difference $>0.2{ }^{\circ} \mathrm{C}$ were $61.8 \pm 19.4$ years, $6.8 \pm 5.3 \mathrm{~cm}$, $5.8 \pm 6.7 \mathrm{~mm}, 0.6 \pm 1.9 \mathrm{mg} / \mathrm{dL}, 4(3-4)$, and $2(2-3)$ respectively. Of tumors with a skin temperature difference $<0.2{ }^{\circ} \mathrm{C}$, the findings were $60.9 \pm 13.8$ years, $5.2 \pm 4.3,9.4 \pm 9.9 \mathrm{~mm}$, $0.5 \pm 1.9 \mathrm{mg} / \mathrm{dL}, 2(1-2)$, and 2 (1-2) respectively. Tumor distance, intertumoral blood supply, and the histological grade
Table 4 Relationship between clinical data and skin temperature differences $\geq 0.2{ }^{\circ} \mathrm{C}$

\begin{tabular}{lllc}
\hline & \multicolumn{2}{l}{$\begin{array}{l}\text { Skin temperature differ- } \\
\text { ences }\left({ }^{\circ} \mathrm{C}\right)\end{array}$} & \\
\cline { 2 - 3 } & $0.2 \leq$ & $<$ value \\
\cline { 2 - 3 } & & & 0.2 \\
Sex $(n)$ & 28 & 37 & \\
Men & 24 & 29 & \\
Women & $61.8 \pm 19.4$ & $60.9 \pm 13.8$ & 0.77 \\
Age (years old) & $6.8 \pm 5.3$ & $5.2 \pm 4.3$ & 0.08 \\
Size (cm) & $5.8 \pm 6.7$ & $9.4 \pm 9.9$ & $0.03 *$ \\
Distance (mm) & $0.6 \pm 1.9$ & $0.5 \pm 1.9$ & 0.7 \\
CRP (mg/dL) & $4[3-4]$ & $2[1-2]$ & $<0.001^{*}$ \\
G grade (median [IQR]) & $2[2-3]$ & $2[1-2]$ & $0.02^{*}$ \\
Histological grade (median & & & \\
$\quad[$ IQR]) & & & \\
\hline
\end{tabular}

$C R P$ C-reactive protein; $G$ grade grades (1-4) classified according to the modified Giovagnorio classification, based on the amount of blood supply in the tumor; $I Q R$ inter-quartile range $* p<0.05$

were found to be associated with skin temperature differences by univariate analysis $(p=0.03, p<0.001$, and $p=0.02$, respectively). Using logistic regression analysis, only intertumoral blood supply was found to be associated with a difference in skin temperature $\geq 0.2{ }^{\circ} \mathrm{C}$ (OR 3.22, 95\% CI 2.03-5.13, $p<0.001$; Table 5). Table 6 shows the distribution of intertumoral blood flow grades in benign and malignant tumors. It can be seen that Grade 4 blood flow was more common than grade 1 in the malignant tumor group. The average value of the difference in skin temperature for each grade was $0.029 \pm 0.27$ (grade 1), $0.026 \pm 0.18$ (grade 2), $0.14 \pm 0.33$ (grade 3 ), and $0.53 \pm 0.43$ (grade 4 ). The average value of the difference in skin temperature was significantly higher in grade 4 than in other grades $(p<0.001)$ (Fig. 5).

\section{The relationship between tumor-related skin temperature and SUVmax using PET-CT imaging}

Table 7 shows the relationship between SUVmax values and skin temperature differences of $0.2{ }^{\circ} \mathrm{C}$. Seventeen patients with soft-tissue tumors had a skin temperature difference $>0.2^{\circ} \mathrm{C}$, and 9 patients had tumors with skin temperature differences $<0.2{ }^{\circ} \mathrm{C}$, with average SUVmax values of 7.4 and 4.6, respectively. There was no correlation between SUVmax and $0.2^{\circ} \mathrm{C}$ skin temperature differences $(p=0.23)$.

\section{Discussion}

In this study, a statistically significant difference was found in tumor-related temperature differences in the healthy and affected sides of benign lesions and malignant tumors in 118 
Table 5 Factors contributing to skin temperature differences $\geq 0.2{ }^{\circ} \mathrm{C}$ in softtissue tumors

\begin{tabular}{|c|c|c|c|c|}
\hline & \multirow[t]{2}{*}{$p$ value } & \multirow[t]{2}{*}{ OR } & \multicolumn{2}{|c|}{$95 \% \mathrm{CI}$} \\
\hline & & & Lower & Upper \\
\hline Sex (male versus female) & 0.89 & 1.07 & 0.42 & 2.71 \\
\hline Age (for 1-year increments) & 0.63 & 0.99 & 0.97 & 1.02 \\
\hline Tumor size (for $0.1-\mathrm{cm}$ increments) & 0.78 & 1.02 & 0.92 & 1.13 \\
\hline Tumor depth (for 0.01-mm increments) & 0.09 & 0.95 & 0.89 & 1.01 \\
\hline The value of CRP (for $0.01-\mathrm{mg} / \mathrm{dL}$ increments) & 0.61 & 0.95 & 0.77 & 1.17 \\
\hline G grade (for 1-grade increments) & $<0.001 *$ & 3.22 & 2.03 & 5.13 \\
\hline
\end{tabular}

$C I$ confidence interval; $C R P$ C-reactive protein; $G$ grade grades (1-4) classified according to the modified Giovagnorio classification, based on the amount of blood supply in the tumor; OR odds ratio

$* p<0.05$
Table 6 Pathological diagnosis and intertumoral blood supply

\begin{tabular}{llllll}
\hline & \multicolumn{3}{l}{ G grade } & & Total \\
\cline { 2 - 5 } & 1 & 2 & 3 & 4 & \\
\hline Malignant $(n)$ & 7 & 11 & 11 & 28 & 57 \\
Benign $(n)$ & 28 & 16 & 14 & 3 & 61 \\
Total $(n)$ & 35 & 27 & 25 & 31 & 118 \\
\hline
\end{tabular}

$G$ grade grades (1-4) classified according to the modified Giovagnorio classification based on the amount of blood supply in the tumo;. $n$ number

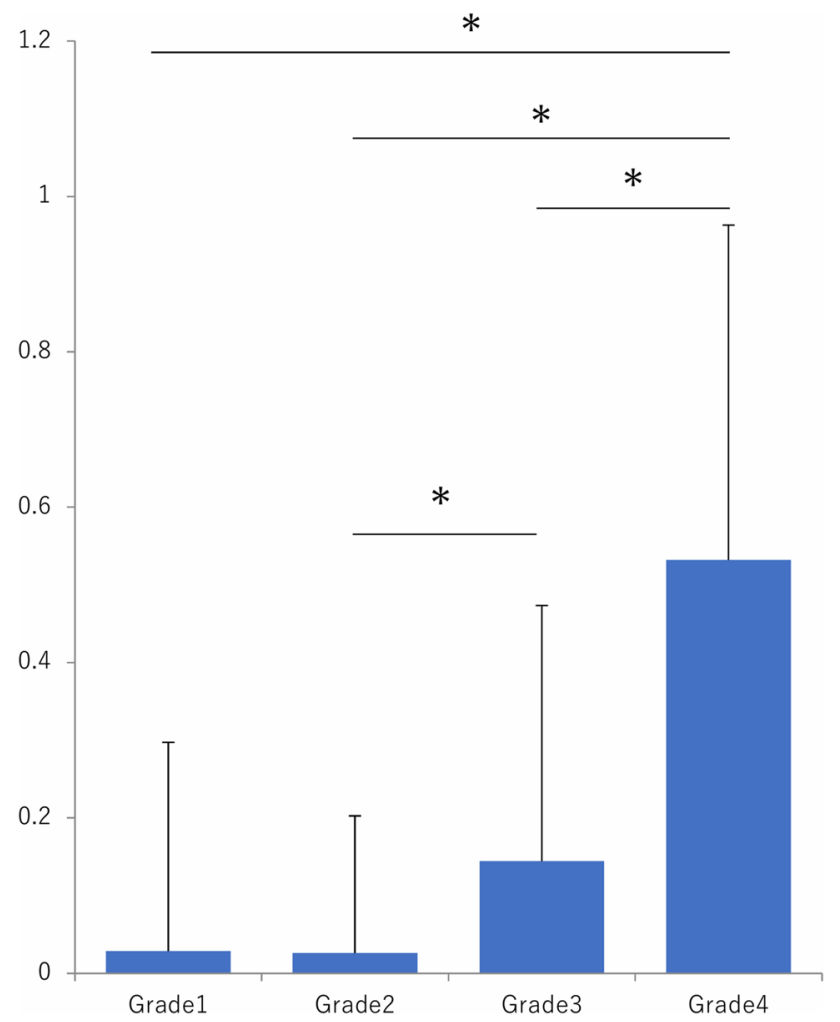

Fig. 5 Average values of the difference in skin temperature for each intertumoral blood flow grade. The vertical axis shows the value of the difference in skin temperature. ${ }^{*} p<0.001$ soft-tissue tumors, excluding adipose tumors. Using a ROC curve, a cut-off value of $0.2^{\circ} \mathrm{C}$ was considered a meaningful index for the skin temperature difference. The factors contributing to the skin temperature differences were unrelated to the size and depth of the tumor and the CRP level; however, they were related to the amount of intertumoral blood supply, as measured using echo-color Doppler. There was no association between the difference in skin temperature and SUVmax in FDG-PET scans of soft-tissue tumors.

Palpation is considered important in the diagnosis of soft-tissue tumors. Palpatory findings in soft-tissue tumors include hardness, swelling, mobility, and temperature. We considered that various factors might be related to elevated temperature in malignant tumors; therefore, we focused on detecting temperature through palpation. Skin temperature is generally compared between healthy and affected sides; however, this is a highly subjective assessment, and an objective data assessment is required. Therefore, we conducted this study using a thermography camera to determine whether malignant soft-tissue tumors had an elevated skin temperature compared to benign soft-tissue lesions. Based on the previous reports, we determined that 20 samples would be sufficient to verify inter-observer reliability [17]. Since the value of ICC was 0.9982 , it was shown that the measurement of the skin temperature by the thermography camera was highly reliable. Elevation of tumor-related skin temperature is meaningful in predicting tumor malignancy. Further, we considered that identifying clinical factors related to tumors that elevate skin temperature might help to predict tumor grade. Therefore, we examined clinical data to identify factors that elevate the skin temperature of malignant soft-tissue tumors. Clinical parameters included sex, age, tumor size and distance, the inflammatory marker CRP, intertumoral blood supply, histological grade, and glucose metabolism. Intertumoral blood supply was assessed using echo-color Doppler, and glucose metabolism was assessed using PET-CT scans according to the SUVmax value.

We investigated the relationship between actual skin temperature differences and the presence of malignant 
Table 7 The relationship between skin temperature differences $\geq 0.2{ }^{\circ} \mathrm{C}$ and the SUVmax value

\begin{tabular}{lll}
\hline & \multicolumn{2}{l}{ Skin temperature differences $\left({ }^{\circ} \mathrm{C}\right)$} \\
\cline { 2 - 3 } & $0.2 \leq$ & $<0.2$ \\
\hline SUVmax & $7.4 \pm 6.4$ & $4.6 \pm 2.9$ \\
\hline
\end{tabular}

soft-tissue tumors. Skin temperature differences between healthy and affected sides of a malignant tumor were found to be statistically significantly higher than those of benign lesions in 118 soft-tissue tumors, excluding adipose tumors $(p<0.001)$. It has previously been reported that melanoma exhibits a higher temperature than benign skin lesions [3]. Based on the above, it is clear that malignant tumors generate heat. Tumor size, distance, the presence of inflammation, intertumoral blood flow, histological grade, and glucose metabolism are some of the possible factors involved in heat generation; therefore, the relationship between these factors and tumor-related skin temperature elevation was examined.

CRP is known to be induced as an inflammatory response. It has been reported that tissue inflammation accompanies malignant tumor growth and leads to elevated CRP levels [18]. In addition, Nakamura et al. reported that elevated serum CRP levels identified prior to initial treatment might be a marker of aggressive soft-tissue sarcoma characteristics [19]. In our study, malignant soft-tissue tumors also had statistically significantly higher CRP levels than benign lesions $(p=0.02)$; however, an elevated CRP value did not contribute to skin temperature differences $\geq 0.2{ }^{\circ} \mathrm{C}$.

Malignant tumors are often hypervascular, resulting in temperature elevation [6]. More specifically, an abnormally elevated temperature in the breast can indicate vascular issues or malignancy [20]. A temperature difference that ranges between 1 and $2.5^{\circ} \mathrm{C}$ has been suggested to be a suspicious clinical finding [21]. In this study, we found that skin temperature differences in malignant soft-tissue tumors were often higher than those of benign lesions. ROC curve analysis indicated that the cut-off value for skin temperature differences between benign lesions and malignant tumors was $0.2{ }^{\circ} \mathrm{C}$ (Fig. 4), and skin temperature differences $\geq 0.2{ }^{\circ} \mathrm{C}$ were significantly associated with malignancy $(p<0.001$; Table 3). Previous study results showed that $>50 \%$ of orthopedic oncologists could accurately detect a skin temperature difference of $\geq 0.2^{\circ} \mathrm{C}$ [8]. Therefore, skin temperature differences of $0.2{ }^{\circ} \mathrm{C}$ in soft-tissue tumors could be detected using careful palpation.

Logistic regression analysis was performed to determine whether clinical parameters were affected due to elevated skin temperature (Table 5). The cut-off value for a difference in skin temperature in a malignant tumor was used as the target factor. Our results showed that the amount of intertumoral blood supply was the only factor that increased skin temperature $\left(\geq 0.2{ }^{\circ} \mathrm{C} ; p<0.001\right)$. In breast cancer, it has been reported that differences in heat generation between malignant and healthy tissues can be attributed to differences in blood supply [6]. Moreover, similar to previous studies using echo-color Doppler $[22,23]$, our results showed that malignant tumors had higher intertumoral blood supply than benign lesions $(p<0.001)$. CRP was not found to be a significant factor of tumor-related skin temperature elevation $\geq 0.2{ }^{\circ} \mathrm{C}$ $(p=0.61)$. Tumor size and existing distance were also unrelated to increased skin temperature $>0.2{ }^{\circ} \mathrm{C}(p=0.78$ and $p=0.09$, respectively). Multivariate analysis could not be performed because the number of cases could not be matched, but histological grade was a significant factor of tumor-related skin temperature elevation in univariate analysis $\geq 0.2{ }^{\circ} \mathrm{C}(p=0.02)$.

A tumor's skin temperature has been reported to rise due to hypermetabolism in the surrounding area [7]. Therefore, we hypothesized that increased glucose metabolism contributes to elevated skin temperature. A molecular in vivo imaging technique, namely, 18F-FDG-PET, is used to evaluate patients with cancer. FDG is a glucose analog that tumor cells take up proportionate to their glucose consumption, which is increased following the Warburg effect in the surrounding tissues [24]. Therefore, the relationship between SUVmax and skin temperature elevation in soft-tissue tumors was investigated; however, no significant difference was found in this study $(p=0.23)$. Regarding SUVmax, the sample size was small because the number of patients for whom PET-CT scans had been taken was limited. Therefore, larger sample size may have obtained different results. However, SUVmax with conventional FDG-PET is limited to differentiating benign from malignant soft-tissue masses, including many kinds of histologic subtypes in surrounding areas [25]; therefore, it may be challenging to associate SUVmax values with the diagnosis of soft-tissue tumors.

This study had several limitations. This was a retrospective, single-center study. Prospective studies are needed to further support our findings. Moreover, the small sample size and the types of tumors we assessed varied histologically.

In conclusion, this study was the first study to investigate the relationship between elevated skin temperature and the histopathological diagnosis of soft-tissue tumors, excluding adipose tumors, and to examine clinical factors that increase skin temperature in patients with soft-tissue tumors. A skin temperature difference of $0.2{ }^{\circ} \mathrm{C}$ was used as the cut-off value for suspected malignant tumors. The amount of intertumoral blood supply was a factor influencing elevations in skin temperature. Therefore, a diagnosis of elevated skin temperature using thermography could indicate the presence of malignancy in patients with soft-tissue masses. 
Author contributions Conceptualization, AS and $\mathrm{MH}$; methodology, AS and NT; validation, $\mathrm{NO}$ and $\mathrm{MH}$; formal analysis, AS; investigation, AS, NT, and YB; data curation, $\mathrm{MH}$; writing — original draft preparation, AS; writing - review and editing, $\mathrm{MH}$ and NO.; supervision, $\mathrm{NH}$; project administration, $\mathrm{AS}, \mathrm{MH}$ and $\mathrm{NH}$; all authors have read and agreed to the published version of the manuscript.

Funding No financial support was received for this study.

\section{Declarations}

Conflict of interest The authors have no conflicts of interest to disclose.

Ethical approval This study was approved by Osaka City University Graduate of Medicine (approval number: 4289) and all investigations were conducted in accordance with the Osaka City University Hospital ethical principles of research.

Patient consent for publication The patients and their families provided written consent for the publication of patient data.

\section{References}

1. Kolarić D, Herceg Z, Nola IA et al (2013) Thermographya feasible method for screening breast cancer? Coll Antropol 37:583-588

2. Santa Cruz GA, Bertotti J, Marín J et al (2009) Dynamic infrared imaging of cutaneous melanoma and normal skin in patients treated with BNCT. Appl Radiat Isot 67(7-8 Suppl):S54-58

3. Shada AL, Dengel LT, Petroni GR et al (2013) Infrared thermography of cutaneous melanoma metastases. J Surg Res 182:e9-e14

4. Anbar M (2002) Assessment of physiologic and pathologic radiative heat dissipation using dynamic infrared imaging. Ann N Y Acad Sci 972:111-118

5. Ring EF (1998) Progress in the measurement of human body temperature. IEEE Eng Med Biol Mag 17:19-24

6. Button TM, Li H, Fisher P et al (2004) Dynamic infrared imaging for the detection of malignancy. Phys Med Biol 49:3105-3116

7. Buzug TM, Schumann F, Pfaffmann L et al (2006) Functional infrared imaging for skin-cancer screening. Conf Proc IEEE Eng Med Biol Soc 2006:2766-2769

8. Shimatani A, Hoshi M, Oebisu N et al (2020) Clinical significance of thermal detection of soft-tissue tumors. Int J Clin Oncol 25:1418-1424

9. Araújo MC, Lima RCF, De Souza RM (2014) Interval symbolic feature extraction for thermography breast cancer detection. Expert Syst Appl 41:6728-6737
10. Fernández-Cuevas I, Marins JCB, Lastras JA et al (2015) Classification of factors influencing the use of infrared thermography in humans: a review. Infrared Phys Technol 71:28-55

11. Lawson R (1956) Implications of surface temperatures in the diagnosis of breast cancer. Can Med Assoc J 75:309-311

12. Lahiri BB, Bagavathiappan S, Jayakumar T et al (2012) Medical applications of infrared thermography: a review. Infrared Phys Technol 55:221-235

13. Ring F (2010) Thermal imaging today and its relevance to diabetes. J Diabetes Sci Technol 4:857-862

14. Martinez-Jimenez MA, Loza-Gonzalez VM, Kolosovas-Machuca ES et al (2021) Diagnostic accuracy of infrared thermal imaging for detecting COVID-19 infection in minimally symptomatic patients. Eur J Clin Invest 51:e13474

15. Fletcher CDM, Bridge JA, Hogendoorn PCW et al (2002) World Health Organization classification of tumors. Pathology and genetics of tumors of soft tissue and bone. IARC Press, Lyon, pp 341-367

16. Oebisu N, Hoshi M, Leguchi M et al (2014) Contrast-enhanced color Doppler ultrasonography increases diagnostic accuracy for soft tissue tumors. Oncol Rep 32:1654-1660

17. Doros G, Lew R (2010) Design based on intra-class correlation coefficients. Am J Biostat 1(1):1-8

18. Coussens LM, Werb Z (2002) Inflammation and cancer. Nature 420:860-867

19. Nakamura T, Matsumine A, Matsubara T et al (2012) Clinical significance of pretreatment serum C-reactive protein level in soft tissue sarcoma. Cancer 118:1055-1061

20. Gautherie M, Gros CM (1980) Breast thermography and cancer risk prediction. Cancer 45:51-56

21. Bjurstam N, Hedberg K, Hultborn KA et al (1974) Diagnosis of breast carcinoma. An evaluation of clinical examination, mammography, thermography and aspiration biopsy in breast disease. Prog Surg 13:1-65

22. Nagano S, Yahiro Y, Yokouchi M et al (2015) Doppler ultrasound for diagnosis of soft tissue sarcoma: efficacy of ultrasound-based screening score. Radiol Oncol 49:135-140

23. Giovagnorio F, Andreoli C, De Cicco ML (1999) Color Doppler sonography of focal lesions of the skin and subcutaneous tissue. J Ultrasound Med 18:89-93

24. Suárez-Piñera M, Belda-Sanchis J, Taus A et al (2018) FDG PETCT SUVmax and IASLC/ATS/ERS histologic classification: a new profile of lung adenocarcinoma with prognostic value. Am J Nucl Med Mol Imaging 25(8):100-109

25. Aoki J, Watanabe H, Shinozaki T et al (2003) FDG-PET for preoperative differential diagnosis between benign and malignant soft tissue masses. Skeletal Radiol 32:133-138

Publisher's Note Springer Nature remains neutral with regard to jurisdictional claims in published maps and institutional affiliations. 